\title{
BAER AND QUASI-BAER PROPERTIES OF GROUP RINGS
}

\author{
ZHONG YI and YIQIANG ZHOU 9
}

(Received 1 November 2005; revised 1 May 2006)

Communicated by J. Du

\begin{abstract}
A ring $R$ is said to be a Baer (respectively, quasi-Baer) ring if the left annihilator of any nonempty subset (respectively, any ideal) of $R$ is generated by an idempotent. It is first proved that for a ring $R$ and a group $G$, if a group ring $R G$ is (quasi-) Baer then so is $R$; if in addition $G$ is finite then $|G|^{-1} \in R$. Counter examples are then given to answer Hirano's question which asks whether the group ring $R G$ is (quasi-) Baer if $R$ is (quasi-) Baer and $G$ is a finite group with $|G|^{-1} \in R$. Further, efforts have been made towards answering the question of when the group ring $R G$ of a finite group $G$ is (quasi-) Baer, and various (quasi-) Baer group rings are identified. For the case where $G$ is a group acting on $R$ as automorphisms, some sufficient conditions are given for the fixed ring $R^{G}$ to be Baer.
\end{abstract}

2000 Mathematics subject classification: primary 16S34; secondary $16 \mathrm{E} 50$.

\section{Introduction}

Throughout this paper $R$ is assumed to be an associative ring with unity. For a subset $X$ of $R$, let $\mathrm{I}_{R}(X)$ denote the left annihilator of $X$ in $R$. A ring $R$ is said to be a Baer (respectively, quasi-Baer) ring if for any nonempty subset (respectively, any ideal) $X$ of $R$ we have $\mathrm{l}_{R}(X)=R e$ where $e^{2}=e \in R$. The concept of a Baer ring was introduced by Kaplansky in [9] to abstract properties of rings of operators on a Hilbert space, while the notion of a quasi-Baer ring was first used by Clark [5] in 1967 to characterize when a finite dimensional algebra with unity over an algebraically closed field is isomorphic to a twisted matrix units semigroup algebra. The definitions of Baer and quasi-Baer rings are indeed left-right symmetric by [9] and [5]. For the development and an up-to-date account of the study of quasi-Baer and Baer rings, we refer to the article of Birkenmeier, Kim and Park [1].

(C) 2007 Australian Mathematical Society 1446-7887/07 \$A2.00+0.00 
The objective of this paper is to consider the question of when a group ring is (quasi-) Baer. Several related results can be recalled. If $R$ is a quasi-Baer ring and $C_{\infty}$ is the infinite cyclic group and $H$ is the discrete Heisenberg group, then the group rings $R C_{\infty}$ and $R H$ are quasi-Baer. This result was obtained in [2], following the authors' result that a ring $R$ is quasi-Baer if and only if $R[x]$ is quasi-Baer, and if and only if $R\left[x, x^{-1}\right]$ is quasi-Baer. For an ordered monoid $G$, it was proved in Hirano [7] that if $R$ is a quasi-Baer ring then the monoid ring $R G$ is quasi-Baer and that $R G$ is a reduced Baer ring if and only if the same is true of $R$. It was proved in [6] that if $R$ is a reduced ring and $G$ is a so called 'u.p.' semigroup then the semigroup ring $R G$ is Baer if and only if the same is true of $R$. In [3], the authors proved that for a so-called 'u.p.' monoid $G$, the monoid ring $R G$ is quasi-Baer if and only if the same is true of $R$. The main idea in proving all these results is similar to that used in the cases of (Laurent) polynomial rings and it does not help for the question of when a group ring is (quasi-) Baer (which was raised in [1, Question 2.12]). In the Open Problem Section of the Third International Symposium on Ring Theory (Kyongju, South Korea, 1999), Hirano asked whether the group ring $R G$ is quasi-Baer if $R$ is quasi-Baer and $G$ is a finite group with $|G|^{-1} \in R$.

The group ring of a group $G$ over a ring $R$ is denoted by $R G$. Write $C_{n}$ for the cyclic group of order $n$. The following results are obtained: If $R G$ is (quasi-) Baer then so is $R$; if $R G$ is quasi-Baer and $G$ is a finite group then $|G|^{-1} \in R$. As a response to Hirano's question, two integral domains $R_{1}, R_{2}$ with $2^{-1} \in R_{1}$ and $3^{-1} \in R_{2}$ are constructed such that $R_{1} C_{2^{k}}$ and $R_{2} C_{3^{\prime}}$ are not quasi-Baer for any $k \geq 2$ or any $l \geq 1$. We also construct a Baer ring $R$ with $6^{-1} \in R$ such that $R S_{3}$ is not Baer. In addition, we prove that Hirano's question has a positive answer when $G=C_{2}$ or $G=S_{3}$ and that if $D_{\infty}$ is the infinite dihadral group then $R D_{\infty}$ is quasi-Baer if and only if $R$ is quasi-Baer. Two sufficient conditions are obtained for a fixed ring to be Baer.

For any finite subgroup $H$ of a group $G$, we let $\hat{H}=\sum_{h \in H} h$. If $g \in G$ has finite order, we define $\hat{g}=\hat{H}$ where $H=\langle g\rangle$. We write $\mathbb{Z}$ for the ring of integers and $\mathbb{Z}_{n}$ for the ring of integers modulo $n$. As usual, $\mathbb{Q}$ is the field of rationals and $\mathbb{C}$ denotes the field of complex numbers. The imaginary unit is denoted by $\mathbf{i}$. The $n \times n$ matrix ring over $R$ is denoted $\mathbb{M}_{n}(R)$.

\section{Necessary conditions}

We start by proving the following.

THEOREM 2.1. Let $R$ be a subring of a ring $S$ such that both share the same identity. Suppose that $S$ is a free left $R$-module with a basis $G$ such that $1 \in G$ and ag $=g a$ for all $a \in R$ and all $g \in G$. If $S$ is (quasi-) Baer then so is $R$. 
PROOF. We give the proof for the case of quasi-Baer rings and the proof for the case of Baer rings is similar. Let $I$ be an ideal of $R$. Since $S$ is quasi-Baer, $\mathbf{l}_{S}(S I)=S e$ where $e^{2}=e \in S$. Write $e=a_{0} g_{\alpha(0)}+\cdots+a_{n} g_{\alpha(n)}$ where $g_{\alpha(0)}=1$ and the $g_{\alpha(i)} \in G$ are distinct and $a_{i} \in R$. Then for all $a \in I$ we have

$$
0=e a=\left(a_{0} g_{\alpha(0)}+\cdots+a_{n} g_{\alpha(n)}\right) a=a_{0} a g_{\alpha(0)}+\cdots+a_{n} a g_{\alpha(n)},
$$

which shows that $a_{i} a=0$. Therefore $a_{i} I=0$ for $i=0, \ldots, n$. Thus

$$
a_{i} S I=a_{i}\left(\oplus_{g \in G} R g\right) I=a_{i} \sum(R I) g=\sum a_{i} I g=0 .
$$

So $a_{i} \in \mathrm{l}_{S}(S I)=S e$, which implies that $a_{i}=a_{i} e$. It follows that $a_{0}^{2}=a_{0} \in R$.

Because $a_{0} I=0$, we have $R a_{0} \in \mathrm{l}_{R}(I)$. If $r \in \mathrm{l}_{R}(I)$ then

$$
r S I=r\left(\oplus_{g \in G} R g\right) I=r \sum(R I) g=\sum r I g=0 .
$$

So $r \in \mathbf{l}_{S}(S I)=S e$. This shows that

$$
r=r e=r\left(a_{0} g_{\alpha(0)}+\cdots+a_{n} g_{\alpha(n)}\right)=r a_{0} g_{\alpha(0)}+\cdots+r a_{n} g_{\alpha(n)} .
$$

So $r=r a_{0} \in R a_{0}$. Hence $\mathrm{l}_{R}(I)=R a_{0}$.

COROLLARY 2.2. Let $R$ be $a$ ring and $G$ be a group. If the group ring $R G$ is (quasi-) Baer then so is $R$.

PROOF. Note that $S=R G=\oplus_{g \in G} R g$ is a free left $R$-module with a basis $G$ satisfying the assumptions of Theorem 2.1.

COROLLARY 2.3. [2] If $R[x]$ or $R\left[x, x^{-1}\right]$ is (quasi-) Baer then so is $R$.

PROOF. $R[x]$ and $R\left[x, x^{-1}\right]$ are free $R$-modules with bases $\left\{x^{i}: i=0,1, \ldots\right\}$ and $\left\{x^{i}: i=0, \pm 1, \ldots\right\}$ satisfying the assumptions of Theorem 2.1 .

THEOREM 2.4. If $G$ is a finite group and the group ring $R G$ is quasi-Baer then $|G|^{-1} \in R$.

PROOF. It is well known that the augmentation ideal is $\omega(R G)=\sum_{g \in G} R(1-g)$ and $\mathrm{l}_{R G}(\omega(R G))=R G \hat{G}$ (see [11, Lemma 1.2, p.68]). Since $R G$ is quasi-Baer, we have

$$
R G \hat{G}=R G e
$$

where $e^{2}=e \in R G$. There exists $\sum r_{g} g \in R G$ such that $e=\left(\sum r_{g} g\right) \hat{G}=$ $\left(\sum r_{g}\right) \hat{G}$. Thus $\left(\sum r_{g}\right) \hat{G}=e=e^{2}=|G|\left(\sum r_{g}\right)^{2} \hat{G}$, which shows that

$$
\sum r_{8}=|G|\left(\sum r_{8}\right)^{2}
$$


Since $R G \hat{G} \neq 0$, we have $e \neq 0$, so $|G| \neq 0$. Hence the following claim has been proved.

ClAIM 2.5. If a group ring of a finite group is quasi-Baer then the order of the group is not zero in the coefficient ring.

Now let $n=|G|$ and $r=\sum r_{g}$. By (2.1), $\hat{G}=\left(\sum s_{g} g\right) e=\left(\sum s_{g} g\right) r \hat{G}$. Applying augmentation mapping to both sides yields

$$
n=\left(\sum s_{g}\right) r n
$$

By (2.2) and (2.3), it suffices to show that $\mathbf{l}_{R}(n)=0$. Suppose that $\mathbf{l}_{R}(n) \neq 0$. Then $n a=0$ for some nonzero $a \in R$. Thus $n(R a)=R(n a)=0$, so $n \in \mathrm{l}_{R}(R a)$. Since $R$ is quasi-Baer by Corollary $2.2, \mathrm{l}_{R}(R a)=R f$ where $f^{2}=f \in R$. Clearly $f \neq 1$, so $1-f \neq 0$. Moreover, $n(1-f)=0$. But

$$
(1-f)(R G)(1-f)=(1-f) R(1-f) G .
$$

Since $R G$ is quasi-Baer, it follows by Clark [5] that $(1-f)(R G)(1-f)$ is quasi-Baer. Therefore, $S G$ is quasi-Baer where $S=(1-f) R(1-f)$. So $n \neq 0$ in $S$ by the Claim. This contradicts the fact that $n(1-f)=0$. Hence $\mathrm{l}_{R}(n)=0$. The proof is complete.

The next fact is an immediate consequence of Theorem 2.4.

EXAMPLE $1 . \mathbb{Z} G$ is not quasi-Baer for any nontrivial finite group $G$.

EXAMPLE 2. Let $G$ be a finite group and $n$ be an integer with $n>1$. Then the following are equivalent:

(1) $\mathbb{Z}_{n} G$ is Baer.

(2) $\mathbb{Z}_{n} G$ is quasi-Baer.

(3) $\operatorname{gcd}(n,|G|)=1$ and $n$ is square-free.

PROOF. (1) clearly implies (2).

Suppose that (2) holds. Write $n=p_{1}^{s_{1}} \cdots p_{k}^{s_{k}}$ where all $p_{i}$ are prime numbers and $s_{i}>0$. Then $\mathbb{Z}_{n} \cong \mathbb{Z}_{p_{1}^{s_{1}}} \times \cdots \times \mathbb{Z}_{p_{k}^{s_{k}}}$, and $\mathbb{Z}_{n} G \cong \mathbb{Z}_{p_{1}^{s_{1}}} G \times \cdots \times \mathbb{Z}_{p_{k}^{s_{k}}} G$. It follows from (2) that each $\mathbb{Z}_{p_{i}^{s_{i}}} G$ is quasi-Baer. So $\mathbb{Z}_{p_{i}^{s_{i}}}$ is quasi-Baer by Corollary 2.2 and $p_{i}^{s_{i}}$ does not divide $|G|$ by Theorem 2.4. It follows that $s_{i}=1$ and $p_{i}$ does not divide $|G|$. Hence (3) holds.

If (3) is satisfied then $\mathbb{Z}_{n} G$ is a semisimple ring by Maschke's Theorem, so (1) holds. 


\section{Group rings of finite groups and Hirano's question}

Let $R$ be a ring and $G$ be a finite group. If $R G$ is (quasi-) Baer then $R$ is (quasi-) Baer and $|G|^{-1} \in R$. Thus it is natural to ask whether the converse holds true. This question on quasi-Baer rings has been raised by Hirano [8]. In this section, counter-examples to these questions are given and various (quasi-) Baer group rings are identified.

LEMMA 3.1. If $2^{-1} \in R$, then $R C_{2} \cong R \times R$.

PROOF. Write $C_{2}=\{1, g\}$. If $2^{-1} \in R$, then the mapping $R C_{2} \rightarrow R \times R$ given by $a+b g \mapsto(a+b, a-b)$ is a ring isomorphism.

COROLLARY 3.2. If $2^{-1} \in R$, then $R C_{2}$ is (quasi-) Baer if and only if the same is true of $R$.

LEMMA 3.3. If $2^{-1} \in R$ then $R C_{4} \cong R \times R \times R[x] /\left(x^{2}+1\right)$.

PROOF. Write $C_{4}=\left\{1, g, g^{2}, g^{3}\right\}$ and let $e=\left(1+g^{2}\right) / 2$. Since $e$ is a central idempotent of $R C_{4}$, we have $R C_{4}=R C_{4} e \times R C_{4}(1-e)$. Direct calculation shows that $R C_{4} e=\{r e+s g e: r, s \in R\}$ and $R C_{4}(1-e)=\{r(1-e)+s g(1-e): r, s \in R\}$. The mapping $R C_{4} e \rightarrow R[x] /\left(x^{2}-1\right)$ given by $r e+s g e \mapsto r+s \bar{x}$ is a ring isomorphism. Similarly, the mapping $R C_{4}(1-e) \rightarrow R[x] /\left(x^{2}+1\right)$ given by $r(1-e)+s g(1-e) \mapsto r+s \bar{x}$ is a ring isomorphism. Moreover, by Lemma 3.1 $R[x] /\left(x^{2}-1\right) \cong R C_{2} \cong R \times R$.

COROLLARY 3.4. If $2^{-1} \in R$ then $R C_{4}$ is (quasi-) Baer if and only if the same is true of $R[x] /\left(x^{2}+1\right)$.

LEMMA 3.5. Let $R$ be a ring with $3^{-1} \in R$ and $C_{3}=\left\{1, g, g^{2}\right\}$. Then the following statements hold:

(1) $e=\frac{1}{3} \hat{g}$ is a central idempotent of $R C_{3}$ and $R C_{3}=\left(R C_{3}\right) e \times\left(R C_{3}\right)(1-e)$, where $\left(R C_{3}\right) e=\{r e: r \in R\} \cong R$ and

$$
\left(R C_{3}\right)(1-e)=\left\{r+s g+(-r-s) g^{2}: r, s \in R\right\} .
$$

(2) If $R \subseteq \mathbb{C}$ then

$$
\begin{aligned}
R C_{3} & \cong R[x] /\left(x^{3}-1\right) \cong R[x] /(x-1) \times R[x] /\left(x^{2}+x+1\right) \\
& \cong R \times R[x] /\left(x^{2}+x+1\right)
\end{aligned}
$$


PROOF. The verification of (1) is straightforward. If $1 / 3 \in R \subseteq \mathbb{C}$, then the ideals $(x-1)$ and $\left(x^{2}+x+1\right)$ are coprime in $R[x]$, so (2) follows by Chinese Remainder Theorem.

It follows that if $R$ is a subring of $\mathbb{C}$ with $1 / 3 \in R$ then $R C_{3}$ is Baer if and only if the same is true of $R[x] /\left(x^{2}+x+1\right)$.

THEOREM 3.6. Let $R$ be a subring of $\mathbb{C}$ and let $Q(R)$ denote the quotient field of $R$. Consider the polynomial $x^{2}+a x+b \in R[x]$ with $a^{2}-4 b \neq 0$. Let $w$ be a solution of $x^{2}+a x+b=0$ in $\mathbb{C}$. Then $R[x] /\left(x^{2}+a x+b\right)$ is (quasi-) Baer if and only if either $w \in R$ or $R w \cap R=0$ (that is, $w \notin Q(R)$ ).

PROOF. Let $S$ denote the ring $R[x] /\left(x^{2}+a x+b\right)$. Let $x^{2}+a x+b=(x-w)(x-v)$ where $w, v \in \mathbb{C}$. By hypothesis, $w \neq v$. First suppose that $w \notin Q(R)$. Then $S$ is a subring of $\mathbb{C}$ and hence is a domain. In particular, $S$ is Baer. Next suppose that $w \in Q(R)$. Then $v \in Q(R)$. Define the map $\varphi: R[x] \rightarrow Q(R) \times Q(R)$ by $\varphi(f(x))=(f(w), f(v))$. Then the kernel of $\varphi$ is $\left(x^{2}+a x+b\right)$. Hence $S$ can be regarded as a subring of $Q(R) \times Q(R)$. Clearly $S$ is not a domain. We can easily see that $S$ is Baer if and only if $S$ contains the idempotent $(1,0) \in Q(R) \times Q(R)$ and that $(1,0) \in S$ if and only if there exists $r x+s \in R[x]$ such that $r w+s=1$ and $r v+s=0$. Since $x^{2}+a x+b=(x-w)(x-v)$, we deduce that $(a r-1) s=[-(w+v) r-1] s=$ $[-(1-2 s)-1] s=2 s(s-1)=2(-r v)(-r w)=2 r^{2} b$. This implies that $s$ is divisible by $r$ in $R$. Hence $v=-s / r \in R$ and so $w=-a-v \in R$.

Next we give counter-examples to Hirano's question for $G=C_{3}$ and $C_{4}$.

EXAMPLE 3. Let $R_{0}=\left\{n / 2^{k}: n \in \mathbb{Z}, k\right.$ a non-negative integer $\}$. Then $R_{0}$ is a subring of $\mathbb{Q}$. Set

$$
R=\left\{a+3 b \mathbf{i}: a, b \in R_{0}\right\} .
$$

Then $R$ is a subring of $\mathbb{C}$ with $1 / 2 \in R$. Because $R$ is a domain, it is certainly Baer. Clearly $\mathbf{i} \notin R$. Moreover, for $r=3$ and $s=3 \mathbf{i}$, we have $s=r \mathbf{i} \in R \mathbf{i} \cap R$. So, by Theorem 3.6, $R[x] /\left(x^{2}+1\right)$ is not quasi-Baer. Hence $R C_{4}$ is not quasi-Baer by Corollary 3.4.

EXAMPLE 4. Let $R_{0}=\left\{n / 3^{k}: n \in \mathbb{Z}, k\right.$ a non-negative integer $\}$. Then $R_{0}$ is a subring of $\mathbb{Q}$. Set

$$
R=\left\{a+b \sqrt{3} \mathbf{i}: a, b \in R_{0}\right\} .
$$

Then $R$ is a subring of $\mathbb{C}$ with $1 / 3 \in R$. Because $R$ is a domain, it is certainly Baer. Let $a=2 \sqrt{3} \mathbf{i}, b=-(3+\sqrt{3} \mathbf{i})$ and $w=b / a$. Then $a, b \in R$ and $w=(-1+\sqrt{3} \mathbf{i}) / 2$ 
is a root of $x^{2}+x+1$. So $R w \cap R \neq 0$. Moreover, it is easy to verify that the equation $x^{2}+x+1=0$ is not solvable in $R$. Hence it follows by Theorem 3.6 and Lemma 3.5 that $R C_{3}$ is not quasi-Baer.

THEOREM 3.7. If $R G$ is Baer then so is $R H$ for every subgroup $H$ of $G$.

Proof. Let $A$ be a nonempty subset of $R H$. Because $R G$ is Baer and $R H \subseteq R G$, we have $\mathrm{l}_{R G}(A)=R G e$, where $e^{2}=e \in R G$. Write $e=\sum_{h \in H} a_{h} h+\sum_{g \notin H} b_{g} g$. Then for all $\beta \in A$,

$$
0=e \beta=\left(\sum_{h \in H} a_{h} h\right) \beta+\left(\sum_{g \notin H} b_{g} g\right) \beta .
$$

Note that if $h \in H$ and $g \notin H$ then $h g \notin H$. This shows that the support of $\left(\sum_{g \notin H} b_{g} g\right) \beta$ is contained in $G \backslash H$. So it follows by (3.1) that if $\alpha=\sum_{h \in H} a_{h} h$ then $\alpha \in \mathrm{I}_{R H}(A) \subseteq \mathbf{l}_{R G}(A)=R G e$, and hence

$$
\sum_{h \in H} a_{h} h=\left(\sum_{h \in H} a_{h} h\right) e=\left(\sum_{h \in H} a_{h} h\right)^{2}+\left(\sum_{h \in H} a_{h} h\right)\left(\sum_{g \notin H} b_{g} g\right) .
$$

Therefore $\alpha^{2}=\alpha=\alpha e$ and $R H \alpha \subseteq \mathbf{I}_{R H}(A)$. If $\gamma \in \mathbf{I}_{R H}(A)$ then $\gamma A=0$. So $\gamma=\gamma e=\gamma\left(\sum_{h \in H} a_{h} h\right)+\gamma\left(\sum_{g \notin H} b_{g} g\right)$, hence $\gamma=\gamma\left(\sum_{h \in H} a_{h} h\right)=\gamma \alpha$. So $R H \alpha=\mathbf{l}_{R H}(A)$. Hence $R$ is Baer.

EXAMPLE 5. If $R$ is the ring in Example 3 and $G$ is a group containing a subgroup isomorphic to $C_{4}$, then $R G$ is not Baer by Theorem 3.7. In particular, for all $k \geq 2$ the group ring $R C_{2^{k}}$ is not Baer and hence not quasi-Baer. Similarly, if $R$ is the ring in Example 4 and $G$ is a group containing a subgroup isomorphic to $C_{3}$, then $R G$ is not Baer. In particular, for all $k \geq 1$ the group ring $R C_{3^{k}}$ is not quasi-Baer.

LEMMA 3.8. [4, Lemma 4.7] If $6^{-1} \in R$, then $R S_{3} \cong R \times R \times \mathbb{M}_{2}(R)$.

A new family of quasi-Baer rings can be obtained as group rings of $S_{3}$.

COROLLARY 3.9. Let $6^{-1} \in R$. Then $R S_{3}$ is quasi-Baer if and only if the same is true of $R$, and $R S_{3}$ is Baer if and only if the same is true of $M_{2}(R)$.

By Pollingher and Zaks [12, p.134], there exists a Baer ring $R$ such that $6^{-1} \in R$ and $\mathbb{M}_{2}(R)$ is not Baer, so $R S_{3}$ is not Baer.

The next theorem gives another family of quasi-Baer group rings. 
THEOREM 3.10. Let $D_{\infty}=\left\langle x, y: o(x)=2, o(y)=\infty, x y x=y^{-1}\right\rangle$ be the infinite dihedral group. Then $R D_{\infty}$ is quasi-Baer if and only if $R$ is quasi-Baer.

PROOF. The implication in one direction follows from Corollary 2.2. To prove the converse, suppose that $R$ is quasi-Baer. First notice that $R D_{\infty} \cong S[x ; \sigma] /\left(x^{2}-1\right)$ where $S=R\left[y, y^{-1}\right]$ and $\sigma \in \operatorname{Aut}(R)$ with $\sigma(y)=y^{-1}$ and $\sigma(r)=r$ for all $r \in R$ (see $[10, \mathrm{p} .22]$ ). Let $T=S[x ; \sigma] /\left(x^{2}-1\right)$. We next show that $T$ is a quasi-Baer ring. Let $A$ be a nonzero ideal of $T$ and set

$$
\begin{array}{ll}
I=\{a \in S: a+b \bar{x} \in A & \text { for some } b \in S\}, \\
J=\{b \in S: a+b \bar{x} \in A & \text { for some } a \in S\} .
\end{array}
$$

Then $I=J$ is an ideal of $S$. Because $R$ is quasi-Baer, $S$ is quasi-Baer by [2, Theorem 1.2]. Thus $\mathrm{l}_{S}(I)=S e$ where $e^{2}=e \in S$. We verify next that $\mathrm{l}_{T}(A)=T e$. Because $e I=0$, we have $e A=0$, so $T e \subseteq \mathbf{l}_{T}(A)$. Let $c+d \bar{x} \in \mathbf{l}_{T}(A)$ where $c, d \in S$ and let $a_{0} \in I$. Then there exists $b_{0} \in I$ such that $a_{0}+b_{0} \bar{x} \in A$. Therefore, for all $a \in S$, we have

$$
\begin{aligned}
0 & =(c+d \bar{x}) a\left(a_{0}+b_{0} \bar{x}\right)=(c+d \bar{x})\left(a a_{0}+a b_{0} \bar{x}\right) \\
& =\left[c a a_{0}+d \sigma(a) \sigma\left(b_{0}\right)\right]+\left[c a b_{0}+d \sigma(a) \sigma\left(a_{0}\right)\right] \bar{x} .
\end{aligned}
$$

It follows that, for all $a \in S$,

$$
c a a_{0}+d \sigma(a) \sigma\left(b_{0}\right)=0 \text { and } c a b_{0}+d \sigma(a) \sigma\left(a_{0}\right)=0 .
$$

Thus, letting $a=y^{n}(n \in \mathbb{Z})$ yields

$$
c y^{n} a_{0}+d y^{-n} \sigma\left(b_{0}\right)=0 \text { and } c y^{n} b_{0}+d y^{-n} \sigma\left(a_{0}\right)=0 .
$$

Since $y^{n}$ is in the center of $S$, it follows that

$$
y^{2 n} c a_{0}=-d \sigma\left(b_{0}\right) \quad \text { and } \quad y^{2 n} c b_{0}=-d \sigma\left(a_{0}\right) .
$$

Because (3.2) holds for all $n \in \mathbb{Z}$ and because $c, d, a_{0}, b_{0}$ are fixed elements of $S$, we obtain

$$
c a_{0}=d \sigma\left(a_{0}\right)=0 .
$$

Thus $\sigma^{-1}(d) a_{0}=0=c a_{0}$. Since $a_{0}$ is an arbitrary element of $l$, we deduce that $c$ and $\sigma^{-1}(d)$ are in $1_{S}(I)=S e$. Write $c=s_{1} e, \sigma^{-1}(d)=s_{2} e$ where $s_{1}, s_{2} \in S$. Then $d=\sigma\left(s_{2}\right) \sigma(e)$ and so $c+d \bar{x}=s_{1} e+\sigma\left(s_{2}\right) \sigma(e) \bar{x}=\left[s_{1}+\sigma\left(s_{2}\right) \bar{x}\right] e \in T e$. Hence $\mathbf{l}_{r}(A)=T e$ and $T$ is a quasi-Baer ring.

REMARK 1. (1) $R D_{\infty}$ may not be Baer even for an integral domain $R$ : because $\mathbb{Z} C_{2}$ is not Baer (Example 1), it follows, by Theorem 3.7, that $\mathbb{Z} D_{\infty}$ is not Baer.

(2) Since $\mathbb{Z} D_{\infty}$ is quasi-Baer but $\mathbb{Z} C_{2}$ is not, the quasi-Baer analog of Theorem 3.7 does not hold. In Example 8 below, an integral domain $R$ is given such that $R C_{3}$. is not quasi-Baer but $R S_{3}$ is quasi-Baer (so $6^{-1} \in R$ ). 
REMARK 2. In view of Corollary 3.9 and Theorem 3.10, it would be interesting to know when the group ring $R D_{n}$ of the dihedral group $D_{n}$ of order $2 n$ is quasi-Baer. The method used in proving Theorem 3.10 can be used to show that if $R C_{n}$ is quasiBaer and $2^{-1} \in R$ then $R D_{n}$ is quasi-Baer, but the converse does not hold because of Remark 1(2).

\section{Fixed rings}

Let $G$ be a group acting on a ring $R$ as automorphisms and let $R^{G}$ be the fixed ring of $G$ acting on $R$. Here we study the conditions under which $R^{G}$ becomes (quasi-) Baer.

THEOREM 4.1. Let $R$ be a ring and $G$ be a group acting on $R$ as automorphisms such that either (i) $e e^{g}=e^{g} e$ for all $g \in G$ and all $e^{2}=e \in R$ or (ii) $G$ is finite with $|G|^{-1} \in R$. If $R$ is Baer then so is $R^{G}$.

Proof. Let $A$ be a nonempty subset of $R^{G}$. Since $R$ is Baer, we have $\mathbf{l}_{R}(A)=R e$ where $e^{2}=e \in R$. For $g \in G$ we have $R e^{g}=R^{g} e^{g}=(R e)^{g}=\left(\mathbf{l}_{R}(A)\right)^{g}=$ $\mathrm{I}_{R^{g}}\left(A^{g}\right)=\mathrm{I}_{R}(A)=R e$. It follows that

$$
e^{g}=e^{g} e \quad \text { and } \quad e=e e^{g} \quad \text { for all } g \in G .
$$

Suppose that (i) holds. It follows from (4.1) that $e=e^{g}$ for all $g \in G$, so $e \in R^{G}$. Since $e A=0$, we have that $R^{G} e \subseteq \mathbf{I}_{R^{G}}(A)$. For $r \in \mathbf{l}_{R^{G}}(A)$, we have $r A=0$, so $r \in \mathrm{l}_{R}(A)=R e$. Thus, $r=r e \in R^{G} e$. Hence $\mathrm{l}_{R^{G}}(A)=R^{G} e$.

Suppose that (ii) holds. Let $f=(1 /|G|) \sum_{g \in G} e^{g}$. Note that, for all $g, h \in G$, (4.1) implies $e^{h} e^{g}=\left(e^{h} e\right) e^{g}=e^{h}\left(e e^{g}\right)=e^{h} e=e^{h}$. This shows that

$$
\begin{aligned}
f^{2} & =\left(\frac{1}{|G|} \sum_{h \in G} e^{h}\right)\left(\frac{1}{|G|} \sum_{g \in G} e^{g}\right)=\frac{1}{|G|^{2}} \sum_{h \in G} \sum_{g \in G} e^{h} e^{g}=\frac{1}{|G|^{2}} \sum_{h \in G} \sum_{g \in G} e^{h} \\
& =\frac{1}{|G|^{2}} \sum_{h \in G}|G| e^{h}=f .
\end{aligned}
$$

Moreover, $f^{g}=f$ for all $g \in G$. So $f \in R^{G}$. Because $e A=0$ and $f \in \operatorname{Re}$ (by (4.1)), we have $R^{G} f \subseteq \mathbf{l}_{R^{c}}(A)$. Note that $\mathbf{l}_{R^{c}}(A) \subseteq \mathbf{l}_{R}(A)=R e^{g}$ for all $g \in G$. Thus, for $r \in \mathrm{l}_{R^{G}}(A)$, we have $r=r e^{g}$ for all $g \in G$. Hence

$$
r=\frac{1}{|G|}|G| r=\frac{1}{|G|} \sum_{g \in G} r e^{g}=r \frac{1}{|G|} \sum_{g \in G} e^{g}=r f \in R^{G} f .
$$

So $\mathrm{l}_{R^{G}}(A)=R^{G} f$. So $R^{G}$ is Baer. 
The next example shows that the assumptions (i) and (ii) in the previous theorem are necessary.

EXAMPLE 6. [13, Example 6.4] Let $K$ be a field of characteristic $p>0$. Let $R=\mathbb{M}_{2}(K)$ and $G=\langle g\rangle$ where $g: R \rightarrow R, r \mapsto u^{-1} r u$, with $u=\left(\begin{array}{ll}1 & 1 \\ 0 & 1\end{array}\right)$. Then $R$ is Baer (indeed simple Artinian). Directcalculation shows that $R^{G}=\left\{\left(\begin{array}{ll}a & b \\ 0 & a\end{array}\right): a, b \in K\right\}$. So $J\left(R^{G}\right)=\left\{\left(\begin{array}{ll}0 & b \\ 0 & 0\end{array}\right): b \in K\right\}$. If $x=\left(\begin{array}{ll}0 & 1 \\ 0 & 0\end{array}\right)$ then $\mathrm{l}_{R^{G}}(x)=J\left(R^{G}\right)$. Because $J\left(R^{G}\right)$ cannot be generated by an idempotent, $R^{G}$ is not Baer. If $e=\left(\begin{array}{ll}0 & 1 \\ 0 & 1\end{array}\right) \in R$ then $e^{2}=e$ and $e^{g}=\left(\begin{array}{ll}0 & 0 \\ 0 & 1\end{array}\right)$. It is clear that $e e^{g}=e \neq e^{g}=e^{g} e$. Moreover, $|G|=p$ is zero in $R$.

The next example shows that $R$ being Baer is not necessary for $R^{G}$ to be Baer.

EXAMPLE 7. Let $K$ be a field with $2^{-1} \in K$ and $R=\left\{\left(\begin{array}{ll}a & b \\ 0 & a\end{array}\right): a, b \in K\right\}$. Let $g: R \rightarrow R$ be given by $\left(\begin{array}{l}a \\ 0 \\ 0\end{array}\right) \mapsto\left(\begin{array}{cc}a & -b \\ 0 & a\end{array}\right)$, and $G=\langle g\rangle$. It is seen that $R^{G}=\left\{\left(\begin{array}{ll}a & 0 \\ 0 & a\end{array}\right): a \in K\right\} \cong K$. So $R^{G}$ is Baer but $R$ is not quasi-Baer.

In contrast to Theorem 4.1, we give in our concluding example a quasi-Baer ring $S$ and a finite group $G$ acting on $S$ as automorphisms such that $|G|^{-1} \in S$ and $S^{G}$ is not quasi-Baer.

LEMMA 4.2. Suppose that $R$ is a ring with $3^{-1} \in R$. Let $g=(123) \in S_{3}$ and $G=\langle\gamma\rangle$ where $\gamma: R S_{3} \rightarrow R S_{3}$ is given by $\xi \mapsto g^{-1} \xi g$. Then $|G|=3$ and $\left(R S_{3}\right)^{G} \cong R \times R C_{3}$.

Proof. It is clear that $|G|=3$. Let $f=(12)+(13)+(23) \in R S_{3}$. Then

$$
\left(R S_{3}\right)^{G}=\left\{\xi \in R S_{3}: \xi g=g \xi\right\}=\left\{a+b f+c g+d g^{2}: a, b, c, d \in R\right\} .
$$

Let $e=(1 / 3) \hat{g}$. Then $e^{2}=e \in\left(R S_{3}\right)^{G}$ and $e f=f e=f$. So $e$ is a central idempotent of $\left(R S_{3}\right)^{G}$. This shows that

where

$$
\left(R S_{3}\right)^{G}=\left(R S_{3}\right)^{G} e \times\left(R S_{3}\right)^{G}(1-e),
$$

$$
\begin{aligned}
\left(R S_{3}\right)^{G}(1-e) & =\left\{\left(a+b f+c g+d g^{2}\right)(1-e): a, b, c, d \in R\right\} \\
& =\left\{\frac{2 a-c-d}{3}+\frac{-a+2 c-d}{3} g+\frac{-a-c+2 d}{3} g^{2}: a, c, d \in R\right\} \\
& =\left\{r+s g+(-r-s) g^{2}: r, s \in R\right\}, \\
\left(R S_{3}\right)^{G} e & =\left\{\left(a+b f+c g+d g^{2}\right) e: a, b, c, d \in R\right\} \\
& =\{(a+c+d) e+b f: a, b, c, d \in R\}=\left\{r e+\frac{s}{3} f: r, s \in R\right\} \\
& \cong R C_{2} \cong R \times R .
\end{aligned}
$$


The last isomorphism is by Lemma 3.1. To see the second last isomorphism, note that $f^{2}=9 e$ and $f e=e f=f$, so $r e+(s / 3) f \mapsto r+s h$ (where $C_{2}=\{1, h\}$ ) is the required isomorphism. Therefore, it follows by Lemma 3.5 that

$$
\left(R S_{3}\right)^{G} \cong R \times R C_{3} \text {. }
$$

EXAMPLE 8. Let $R_{0}=\left\{n / 6^{k}: n \in \mathbb{Z}, k\right.$ a nonnegative integer $\}$ and set

$$
R=\left\{a+5 b \sqrt{3} \mathbf{i}: a, b \in R_{0}\right\} .
$$

Then $R$ is a subring of $\mathbb{C}$ and $6^{-1} \in R$. It is easy to see that $x^{2}+x+1=0$ is not solvable in $R$. Moreover, if $w=\left(-1 \pm \sqrt{3} \mathbf{i}\right.$ )/2 (a root of $x^{2}+x+1$ ), then $10 w=-5 \pm 5 \sqrt{3} \mathbf{i} \in R$. So $R w \cap R \neq 0$. Hence, by Lemma 3.5 and Theorem 3.6, $R C_{3}$ is not quasi-Baer. Let $G$ be the group in Lemma 4.2. Then $|G|=3$ and $\left(R S_{3}\right)^{G} \cong R \times R C_{3}$ by Lemma 4.2. So it follows that $|G|^{-1} \in R S_{3}$ and $\left(R S_{3}\right)^{G}$ is not quasi-Baer. However, $R S_{3}$ is quasi-Baer by Corollary 3.9. In summary, (1) $R S_{3}$ is quasi-Baer (so $6^{-1} \in R$ ), (2) $R C_{3}$ is not quasi-Baer, (3) $\left(R S_{3}\right)^{G}$ is not quasi-Baer where $|G|=3$ is a unit of $R S_{3}$.

\section{Acknowledgements}

The authors are grateful to the referee who formulated Theorem 3.6 which unifies two situations in the previous version. The research was carried out during a visit by the first author to Memorial University of Newfoundland. He would like to gratefully acknowledge the hospitality received. The work of the first author was supported by China Scholarship Council. The second author was supported by NSERC Grant OGP0194196.

\section{References}

[1] G. F. Birkenmeier, J. Y. Kim and J. K. Park, 'On quasi-Baer rings', in: Algebra and its applications (Athens, OH, 1999), Contemp. Math. 259 (Amer. Math. Soc., Providence, RI, 2000) pp. 67-92.

[2] —_, 'Polynomial extensions of Baer and quasi-Baer rings', J. Pure Appl. Algebra 159 (2001), $25-42$.

[3] G.F. Birkenmeier and J.K. Park, 'Triangular matrix representations of ring extensions', J. Algebra 265 (2003), 457-477.

[4] J. Chen, Y. Li and Y. Zhou, 'Morphic group rings', J. Pure Appl. Algebra 205 (2006), 621-639.

[5] W. E. Clark, 'Twisted matrix units semigroup algebras', Duke Math. J. 34 (1967), 417-424.

[6] N. J. Groenewald, 'A note on extensions of Baer and p.p.-rings', Publ. de L'institut Math. 34 (1983), 71-72.

[7] Y. Hirano, 'On ordered monoid rings over a quasi-Baer ring', Comm. Algebra 29 (2001), 20892095. 
[8] - 'Open problems', in: Proceedings of the 3rd Korea-China-Japan Symposium and the 2nd Korea-Japan Joint Seminar held in Kyongju, June 28-July 3, 1999, Trends Math. (2001) pp. 442.

[9] I. Kaplansky, Rings of Operatcrs, Math. Lecture Notes Series (Benjamin, New York, 1965).

[10] J. C. McConnell and J. C. Robson, Noncommutative Noetherian Rings (John Wiley \& Sons, Chichester, 1987).

[11] D. Passman, The Algebraic Siructure of Group Rings (John Wiley \& Sons, New York-LondonSydney, 1977).

[12] A. Pollingher and A. Zaks, 'On Baer and quasi-Baer rings', Duke Math. J. 37 (1970), 127-138.

[13] Z. Yi, 'Homological dimension of skew group rings and crossed products', J. Algebra 164 (1994), 101-123.

Department of Mathematics, Guangxi Normal University, Guilin, 541004, P.R. China

e-mail: zyi@mailbox.gxnu.edu.cn
Department of Mathematics and Statistics, Memorial University of Newfoundland, St. John's A1C 5S7, Canada e-mail: zhou@math.mun.ca 